\title{
Cognitive improvement following ischemia/reperfusion injury induced by voluntary running-wheel exercise is associated with LncMALAT1-mediated apoptosis inhibition
}

\author{
JIN-LIN SHANG ${ }^{1}$, QING CHENG $^{2}$, SHENG-JIE DUAN ${ }^{1}$, LU LI $^{1}$ and LI-YA JIA ${ }^{1}$ \\ ${ }^{1}$ Neurology Department, Heji Hospital Affiliated to Changzhi Medical College; \\ ${ }^{2}$ Geriatrics Department, Changzhi People Hospital, Changzhi, Shaanxi 046011, P.R. China
}

Received August 1, 2017; Accepted January 19, 2018

DOI: $10.3892 / \mathrm{ijmm} .2018 .3484$

\begin{abstract}
Previous human and animal studies demonstrated that voluntary exercise may improve cognitive function and facilitate neuronal plasticity in ischemia/reperfusion (I/R) models. However, the possible underlying mechanisms remain to be elucidated. Metastasis-associated lung adenocarcinoma transcript 1 (MALAT1), a long noncoding RNA (lncRNA), may be associated with the functions and dysfunctions of endothelial cells. The present study investigated whether spontaneous running-wheel (RW) exercise-induced MALAT1 expression changes may be associated with the cognitive improvement of mice following I/R injury. The expression of MALAT1 was evaluated using reverse transcription-quantitative polymerase chain reaction. Artificial MALAT1 and MALAT1 lentiviral mall interfering (siRNA) were used to alter MALAT1 expression levels in vivo. The Morris Water Maze test was performed to evaluate spatial learning and memory retention in the mice. Changes in the apoptotic rates of hippocampal neurons and levels of apoptosis-associated proteins were also detected. The data revealed that MALAT1 increased in the hippocampus of mice in the RW-treated I/R group and that this was associated with neurological, learning and memory improvement, reduced infarction volumes, decreased apoptosis and alterations to expression levels of apoptosis-associated proteins. Following RW training in I/R-injured mice, lentiviral MALAT1 siRNA conduction partially attenuated the protections induced by voluntary RW. However, exogenous MALAT1 treatment increased the protection. The current findings suggested that voluntary RW protected hippocampal neurons from I/R injury and promoted
\end{abstract}

Correspondence to: Dr Jin-Lin Shang, Neurology Department, Heji Hospital Affiliated to Changzhi Medical College, 271 Taihang East Street, Changzhi, Shaanxi 046011, P.R. China

E-mail: jinlinshang139@126.com

Key words: ischemia/reperfusion, spontaneous running-wheel, MALAT1, learning and memory, apoptosis cognitive restoration, which was associated with lncRNA MALAT1-mediated apoptosis inhibition.

\section{Introduction}

Ischemic brain injury, or ischemic stroke, is a major cause of mortality and disability worldwide (1). Rapid revascularization of the occluded vessels, followed by timely reperfusion, is considered one of the most effective approaches to cerebral ischemic injury treatment. However, this therapy method may evoke subsequent ischemia-reperfusion (I/R) injury. $\mathrm{I} / \mathrm{R}$ damage is an important event in each case and includes the restoration of blood flow following a critical duration of ischemia, which is the primary cause of post-ischemic tissue injury $(2,3)$. I/R injury also induces cognitive deficit, which significantly affects the quality of life of patients who have had a stroke. However, the therapeutic efficacy remains unsatisfactory.

A previous study revealed that cerebral I/R injury differentially changed the expression of numerous noncoding RNAs, termed micro RNAs (miRNAs), upregulating the expression of specific genes and downregulating the level of others (4). Long non-coding RNAs (lncRNAs) are not responsible for protein coding or translation. As a class of key regulatory RNAs, lncRNAs have important roles in various biological processes in mammals, including genomic imprinting, dose compensation, gene regulation, chromatin organization and alternative splicing (5). Furthermore, lncRNAs have been previously identified to be involved in various human diseases. A pervious study revealed that a large number of genomes produced IncRNAs of in humans or mice; however, their effect and function remain to be elucidated (6). LncRNAs are a demonstrated class of novel key regulators in cerebrovascular endothelial pathologies that arise following ischemic injury, such as stroke (7). According to a previous study, ischemic stroke induces expressional changes in both 1ncRNA genes and protein-coding genes, which suggests that the so-called 'stroke-responsive' lncRNAs may have roles in plasticity post ischemic injury (8).

It has been previously reported that a nuclear-residing lncRNA, metastasis-associated lung adenocarcinoma transcript 1 (MALAT1), is involved in the proliferation and 
survival of cancer cells, as well as in tumor metastasis (9). MALAT1 is also enriched in endothelial cells, serving a crucial role in maintaining endothelial cell functions. The abnormal expression of MALAT1 is associated with endothelial cell dysfunction, including inflammatory processes induced by hyperglycemia, angiogenesis and proliferation (9-11). However, there are limited reports regarding the potential role of MALAT1 in I/R injury.

Evidence obtained from both human and animal studies demonstrates that voluntary exercise may improve cognitive function and facilitate neuronal plasticity in I/R-models $(4,12)$. As a rehabilitation strategy, voluntary wheel-running is a demonstrated effective intervention for facilitating motor recovery (13). However, the underlying molecular and epidemic mechanisms remain to be elucidated. The present study investigated the possible effects of MALAT1 on voluntary running-wheel (RW) exercise-induced cognitive improvement following I/R injury.

\section{Materials and methods}

Animal I/R models and spontaneous exercise. A total of 56 male $\mathrm{C} 57 \mathrm{BL} / 6 \mathrm{~J}$ mice with an average weight of $28.31 \pm 2.19 \mathrm{~g}$, aged 4 to 4.5 months, were purchased from the Changzhi Medical College. Animal use and care were in accordance with the animal care guidelines, which conformed to the Guide for the Care and Use of Laboratory Animals, published by the US National Institutes of Health (NIH Publication No. 85-23, revised 1996). All protocols performed in the current study were approved by the Changzhi Medical College Administrative Committee of Experimental Animal Care and Use (license no. cz08329).

Prior to the experiments, the mice were allowed to acclimate to their environment for 1 week. A total of 14 mice underwent a sham-operation and were used as the sham group. For the I/R injury model, 28 mice were subjected to $45 \mathrm{~min}$ of ischemia followed by reperfusion, as previously described (4). Overall, 7 sham-operated mice and 35 I/R-injured mice were separately housed, each in cages equipped with a RW (sham-runners, $\mathrm{n}=7$; I/R-runners, $\mathrm{n}=35)$. The remaining untreated mice served as the sedentary control group (I/R non-runners, $n=7$; sham non-runners, $n=7)$, and were housed in cages with an immobilized RW, as previously described $(14,15)$. All mice used in this work were bred at Changzhi Medical College in a restricted access, temperature-controlled (at $22^{\circ} \mathrm{C}$ ) animal central on a 12-h light/dark cycle, food and water ad libitum. To investigate the potential roles of MALAT1 involved in spontaneous-RW-induced neuroplasticity, the I/R-runners were sub-divided into five groups (I/R-runners, $n=7$; I/R-runners + MALAT1 siRNA, n=7; I/R-runners + MALAT1 scrambled siRNA, $n=7$; I/R-runners + MALAT1, $n=7$; I/R-runners + blank control, $\mathrm{n}=7)$. RWs with a diameter of $12 \mathrm{~cm}$ and a width of $5 \mathrm{~cm}$ (Nalge Nunc International, Rochester, NY, USA) were used to equip the cages for I/R-runners. The number of revolutions was monitored and recorded using Vital Viewer Data Acquisition System version V-4000 (Mini Mitter, Sunriver, OR, USA). Given that $7 \mathrm{pm}$ to $7 \mathrm{am}$ is the most active period for rodents, the average number of revolutions was calculated for each night during this period. The mice were allowed unlimited access to the RWs in their individual cages.
Focal cerebral I/R operation. During surgery, 5\% isoflurane was used to induce anesthesia, and 1-2\% isoflurane was used for maintenance. Focal ischemic injury was generated as described previously $(16,17)$. After $45 \mathrm{~min}$ of ischemia, reperfusion was achieved by permanently withdrawing the suture, as previously described (4).

Neurological deficits evaluation. The neurological deficits of mice were assessed at 1,2 and 3 days post-operation (dpo). Focal neurological scores (FNS), using a 28-point scale, were used to assess neurological deficits, as previously described (18). The total score for each animal ranged from 0-28, the higher scores indicate a greater impairment (18).

MALAT1/MALAT1 siRNA treatment. By transducing with synthetic MALAT1 or siRNA-targeted MALAT1, MALAT1 expression levels were upregulated or downregulated, respectively. Mus musculus (Mmu)-MALAT1, MALAT1 siRNA and scrambled siRNA were designed and purchased from Applied Biological Materials Inc. (Richmond, BC, Canada). I/R-runners received various treatments via intraperitoneal injection with mmu-MALAT1 (10 nmol/20 g body weight, I/R-runners + MALAT1, n=7), MALAT1 blank control (I/R-runners + MALAT1 blank, n=7), mmu-MALAT1 siRNA (5 nmol/20 g body weight, I/R-runners + MALAT1 siRNA, $\mathrm{n}=7$ ), or scrambled siRNA (I/R-runners + MALAT1 scrambled siRNA, $\mathrm{n}=7), 5 \mathrm{~h}$ after I/R injury and twice per week prior to sacrifice (15).

Morris water maze (MWM) test. At 25-30 days after I/R injury, induced immediately followed running, the MWM test was used to evaluate spatial learning and memory, as described previously $(15,19)$. Each mouse underwent the trial thrice daily for six consecutive days. The percentages of time, the path taken in the target quadrant, and the number of target platform crossings were recorded and analyzed using TOPSCAN ${ }^{\mathrm{TM}}$ version 2.0 (Clever Sys, Inc., Reston, VA, USA).

Triphenyl tetrazolium chloride (TTC) staining. TTC staining was performed for infarct volume assessments, as previously described (20). Briefly, coronal sections of the brain, 2-mm thickness, were incubated with $2 \%$ TTC at $37^{\circ} \mathrm{C}$ for $30 \mathrm{~min}$ with gentle agitation. The sections were then fixed at $22-25^{\circ} \mathrm{C}$ in $10 \%$ formalin with PBS for $15 \mathrm{~min}$, and subjected to serial staining steps. Finally, the stained slices were collected for imaging. The infarct volume was quantified and analyzed by Leica image software DMI6000B (Leica Microsystems, Wetzlar, Germany).

Reverse transcription-quantitative polymerase chain reaction $(R T-q P C R)$. The level of MALAT1 in the cerebral cortex and the hippocampus from sham and I/R injured mice was determined using RT-qPCR as previously described (7). Total RNA was isolated using TRIzol ${ }^{\circledR}$ (Thermo Fisher Scientific, Inc., Waltham, MA, USA), according to the manufacturer's protocol. cDNA was synthesized by using Oligo $(\mathrm{dT})^{18}$ and MMLV reverse transcriptase (Promega Corporation, Madison, WI) at $22-25^{\circ} \mathrm{C}$. The iQ SYBR ${ }^{\circledR}$ Green supermix (Bio-Rad Laboratories, Inc., Hercules, CA, USA) was used for qPCR detection, according to the manufacturer's protocol. GAPDH 
served as the endogenous control. LncRNAs expression levels were normalized by calculating the lncRNAs to GAPDH expression ratio $\left(2^{-\Delta \Delta C q}\right)(21)$. qPCR amplification was performed at $95^{\circ} \mathrm{C}$ for $3 \mathrm{~min}$, followed by 40 cycles at $95^{\circ} \mathrm{C}$ for $15 \mathrm{sec}$ and $62^{\circ} \mathrm{C}$ for $60 \mathrm{sec}$. The primer sequences were as follows: GAPDH, forward: 5'-CGAGATCCCTCCAAA ATCAA-3' and reverse: 5'-TTCACACCCATGACGAACAT-3'; MALAT1, forward 5'-GTGATGCGAGTTGTTCTCCG-3' and reverse 5'-CTGGCTGCCTCAATGCCTAC-3' (7,22).

Cell apoptosis assay. Hippocampal tissues were fixed at $22-25^{\circ} \mathrm{C}$ in $4 \%$ formalin with PBS for $30 \mathrm{~min}$, and subjected to serial staining steps. Apoptosis within the hippocampal tissues was evaluated using a TUNEL-POD In Situ apoptosis detection kit (Roche Molecular Systems, Inc., Pleasanton, CA, USA), according to the manufacturer's protocol. The TUNEL-positive/apoptotic cells, as detected by DAB staining at $22-25^{\circ} \mathrm{C}$ for $5-10 \mathrm{~min}$ (Beyotime Institute of Biotechnology, Shanghai, China), were presented as a percentage of the total cells. A phase contrast microscope (Leica) was used for imaging at x40 magnification and 5 fields of view were used. Three independent experiments were performed.

Western blot analysis. Western blotting was used to determine the expression of cytokines associated with apoptosis, which may have been altered by spontaneous RW in I/R injured mice. Antibodies against B cell leukemia/lymphoma 2 (Bcl-2), BCL2-associated X (Bax), BCL2 associated agonist of cell death (Bad), B-cell lymphoma-extra large (Bcl-xL), caspase-3/-8, and GAPDH were purchased from Cell Signaling Technology, Inc. (Danvers, MA, USA). Tissues were homogenized on ice in a RIPA lysis buffer (P0013C; Beyotime Institute of Biotechnology) and centrifuged at 4,500 x $\mathrm{g}$ for 25 min. Protein concentration was assayed with BCA reagent (Sigma-Aldrich; Merck Millipore, Darmstadt, Germany). Total $50 \mathrm{mg}$ cell lysate protein per lane extracts from each cell lines were separated using 15\% SDS-PAGE and transferred to nitrocellulose membranes. The membranes were then blocked with phosphate-buffered saline containing $0.05 \%$ Tween-20 (PBST) with 10\% non-fat dry milk (Sigma-Aldrich; Merck Millipore) overnight at $4^{\circ} \mathrm{C}$ for $12 \mathrm{~h}$, then the membrane was washed three times for $10 \mathrm{~min}$ each time. This was followed by incubation with anti-Bad (1:800; cat. no. AB008; Beyotime Institute of Biotechnology), anti-Bcl-2 (1:500; cat. no. AB112; Beyotime Institute of Biotechnology), anti-Bax (1:500; cat. no. ab32503; Abcam, Cambridge, UK), anti-Bcl-xL (1:1,000; cat. no. ab32370; Abcam), and anti-caspase-3 (1:1,000; cat. no. ab13847; Abcam) and anti-caspase-8 (1:500; cat. no. ab25901; Abcam) primary antibodies at $4^{\circ} \mathrm{C}$ overnight. After washing 3 times with PBST for 10 min each, the membrane was incubated with a horseradish peroxidase-conjugated goat anti-rabbit IgG (1:2,500; cat. no. A0208; Beyotime Institute of Biotechnology) for $2 \mathrm{~h}$ at room temperature. The blots were then subjected to enhanced chemiluminesence-based detection (Beyotime Institute of Biotechnology) as previously described $(23,24)$. The semi-quantitated target proteins were analyzed for each group by using Bio-Rad Gel Imagining system (ChemiDoc ${ }^{\mathrm{TM}}$ XRS+; Bio-Rad Laboratories, Hercules, CA, USA) $(23,24)$.
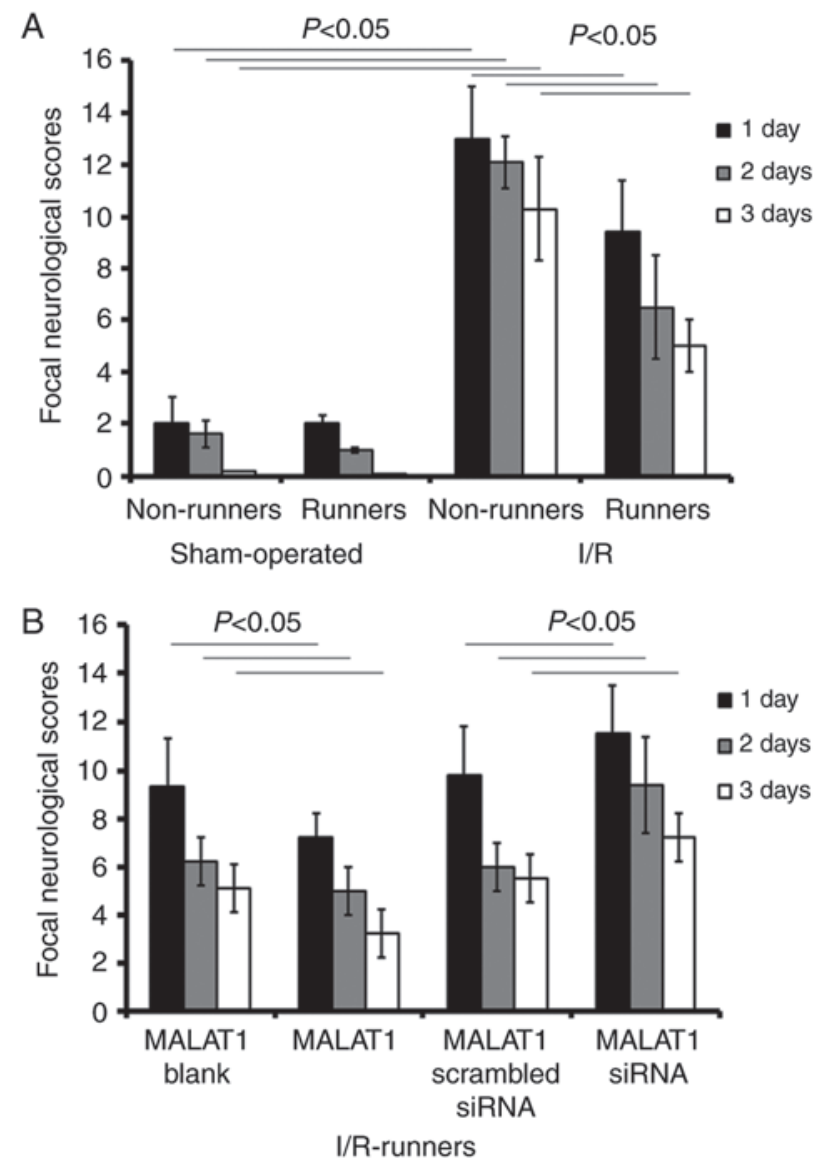

Figure 1.Focal neurological scores evaluation. Neurological deficit evaluations were performed at 1,2 and 3 days post I/R injury. (A) Neurological deficits in sham-operated and I/R injured mice with or without running-wheel exercise, determined via focal neurological scores evaluation. (B) Neurological deficits in I/R-runners treated with exogenous MALAT1 or MALAT1 siRNA. Data are presented as the mean \pm standard deviation. d, days post operation; siRNA, small interfering RNA; MALAT1, metastasis-associated lung adenocarcinoma transcript $1 ; \mathrm{I} / \mathrm{R}$, ischemia/reperfusion.

Statistical analysis. Values are presented as the mean \pm standard deviation. FNS were analyzed using the Kruskal-Wallis test, followed by the Mann-Whitney U test with Bonferroni's correction. Significant differences between the groups were determined using Student's t-test. Two-way repeated-measures ANOVA, followed by Tukey's test, was used for the MWM test analysis. SPSS version 19.0 (IMB Corporation, Armonk, NY, USA) was used for statistical analysis. $\mathrm{P}<0.05$ was considered to indicate a statistically significant difference.

\section{Results}

Neurological scores. The data from the present study revealed that voluntary RW partially rescued neurological impairment, whereas siRNA targeting MALAT1 neutralized that neuroprotection. I/R surgery lead to increased scores relative to the sham operation (I/R Non-runners vs. sham non-runners; $\mathrm{P}<0.05$; Fig. 1). RW exercise significantly reduced neurological scores from 1-3 dpo (I/R Non-runners vs. I/R-runners; $\mathrm{P}<0.05$; Fig. 1). Compared with the I/R-runners + MALAT1 blank controls, MALAT1 treatment significantly decreased neurological scores from 1-3dpo (I/R-runners + MALAT1 


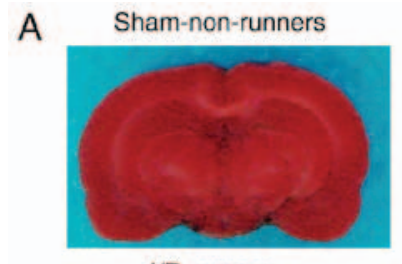

1/R-runners+

MALAT 1 blank

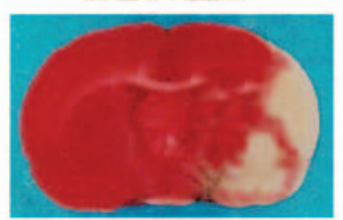

Sham-runners

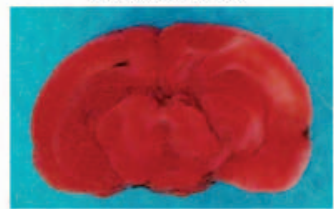

1/R-runners+

MALAT1

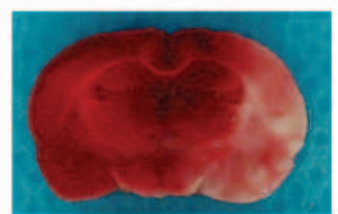

I/R-non-runners

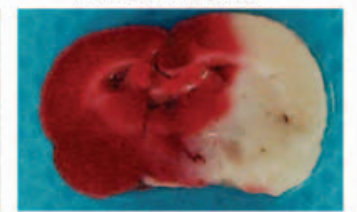

1/R-runners+

MALAT1 scrambled

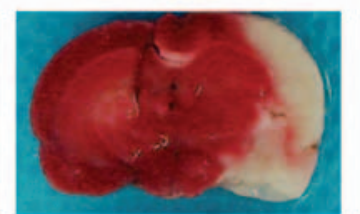

1/R-runners

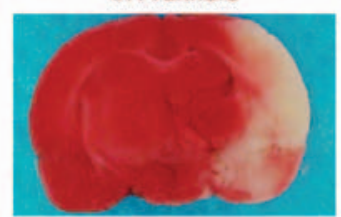

1/R-runners+

MALAT1 SIRNA

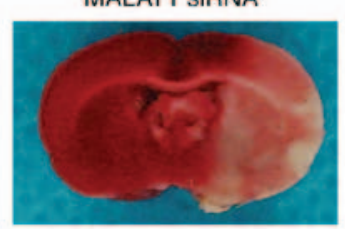

B
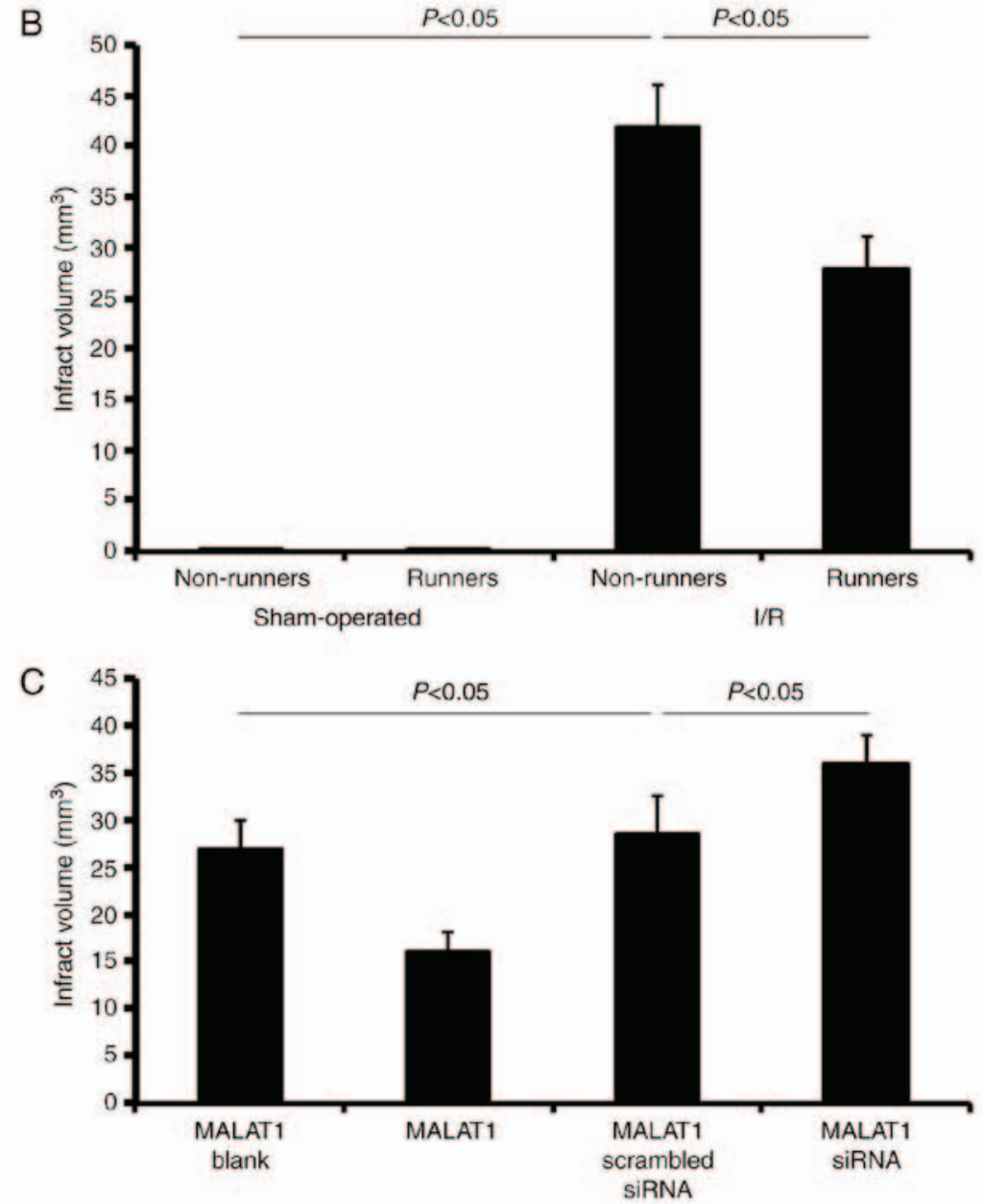

I/R-runners

Figure 2. Infarct volume evaluations in different groups. (A) Infarct volume was detected by triphenyl tetrazolium chloride staining. Quantification of infarct volumes in (B) runners and non-runners and (C) I/R-runners treated with exogenous MALAT1 or siRNA MALAT1. Data are presented as the mean \pm standard deviation. siRNA, small interfering RNA; MALAT1, metastasis-associated lung adenocarcinoma transcript 1; I/R, ischemia/reperfusion.

vs. I/R-runners + MALAT1 blank; P $<0.05$; respectively). Conversely, scores were significantly reduced from 1-3dpo in I/R-runners receiving MALAT1 siRNA treatment compared with the MALAT1 scrambled siRNA group (I/R runners + MALAT1 siRNA vs. I/R runners + MALAT1 scrambled siRNA, P<0.05; Fig. 1).

Infarct volume evaluation. TTC staining revealed no observable damage in the cerebrums of mice subjected to a sham operation (Fig. 2). I/R injuries led to a large infarction, which was attenuated by voluntary RW following the injury (I/R non-runners vs. I/R runners; $\mathrm{P}<0.05$; Fig. 2). Treatment with MALAT1 increased the neuroprotective effects induced by RW following I/R injury (I/R runners + MALAT1 vs. I/R runners + MALAT1 blank control; P $<0.05$; Fig. 2), where MALAT1 siRNA administration partially neutralized these effects (I/R runners + MALAT1 siRNA vs. I/R runners+MALAT1 scrambled siRNA; P<0.05; Fig. 2). 
Changes to MALAT1 levels in different groups. Following I/R injury, MALAT1 expression levels were significantly reduced in the I/R non-runners, compared with in the sham non-runners $(\mathrm{P}<0.05)$. There were no significant differences between sham-operated runners and the non-runners (sham non-runners vs. sham-runners; $\mathrm{P}>0.05$ ). Spontaneous RW increased the expression levels of MALAT1 in I/R injured mice. The expression levels of MALAT1 increased following voluntary RW training in I/R-runners (I/R non-runners vs. I/R runners, $\mathrm{P}<0.05$, Fig. 3).

MALAT1-treatment of I/R-runners induced significant upregulation of MALAT1 expression in the I/R-runners + MALAT1 group vs. I/R-runners + MALAT1 blank $(\mathrm{P}<0.05)$. siRNA targeting MALAT1 effectively reduced MALAT1 expression levels in I/R-runners, and in MALAT1 siRNA-treated mice vs. I/R-runners + MALAT1 scrambled siRNA $(\mathrm{P}<0.05)$. The MALAT1 levels showed no significant difference between the two matched control groups (I/R-runners + MALAT1 blank vs. I/R-runners + MALAT1 scrambled siRNA, P>0.05, Fig. 3).

Spatial learning and memory alterations. The present study used the MWM test to assess the spatial learning of mice subjected to various treatments. During the initial 5 training days, spatial learning loss was assessed by a hidden platform test. The mice in sham-operated groups showed no significant cognitive deficiency. Voluntary RW had no effect on the cognitive changes in sham-operated mice, but induced obvious improvements in spatial learning and memory in $\mathrm{I} / \mathrm{R}$ injured mice. There was no significant difference in the escape latencies between sham non-runners and sham-runners ( $\mathrm{P}>0.05$; Fig. 4A). I/R non-runners appeared to take longer to find the hidden platform compared with I/R-runners $\left(\mathrm{F}_{1,7}=16.35 ; \mathrm{P}<0.01\right.$; Fig. 4A).

Following MALAT1 treatment, I/R-runners had significantly longer escape latencies compared with mice from the I/R-runners + MALAT1 group vs. I/R-runners + MALAT1 blank $\left(\mathrm{F}_{1,7}=7.62\right.$; $\mathrm{P}<0.05$; Fig. 4B). The latency differences were appeared significantly at the third training day (Tukey's test; $\mathrm{P}<0.05$; Fig. 4B). Conversely, MALAT1 siRNA partially neutralized the cognitive improvement induced by spontaneous RW exercise. MALAT1 siRNA-treated I/R mice took longer to find the hidden platform, compared with MALAT1 scrambled siRNA treated ones $\left(\mathrm{F}_{1,7}=13.28 ; \mathrm{P}<0.01 ;\right.$ Fig. $\left.4 \mathrm{~B}\right)$. However, no significant difference was identified in the escape latencies between the two control groups (I/R-runners + MALAT1 blank vs. I/R-runners + MALAT1 scrambled siRNA; P $>0.05$; Fig. 4B). The swimming traces at the fifth training day for mice in different groups are presented in Fig. 4C.

Following the hidden platform test, a probe test was performed to evaluate spatial retention on the sixth day. Sham-runners exhibited no obvious differences when compared with sham-Non-runners for any of the tested parameters $(\mathrm{P}>0.05$; Fig. 4). However, sham non-runners spent longer in the target quadrant compared with the I/R non-runners ( $\mathrm{P}<0.05$, Fig. 4D). Voluntary RW induced notable improvement in memory retention. For I/R-runners, the time spent and number of paths travelled in the target quadrant, as well as the number of target crossings, was increased, compared with I/R non-runners $(\mathrm{P}<0.05$, Fig. 4). Synthetic
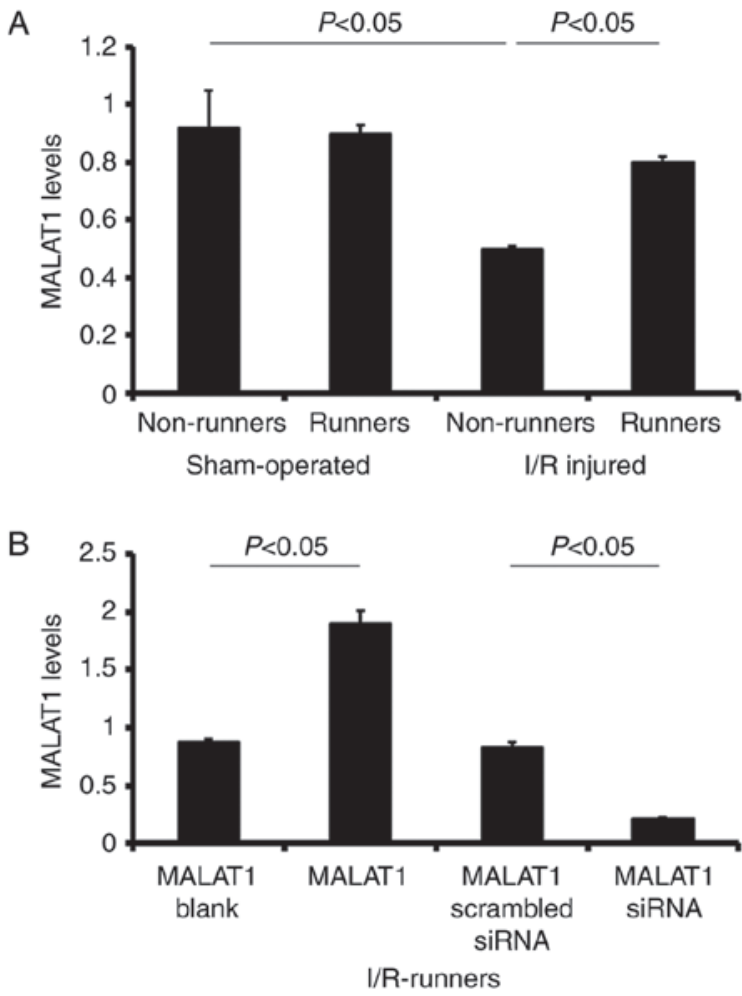

Figure 3. MALAT1 expression in different groups, as detected by reverse transcription-quantitative polymerase chain reaction. (A) MALAT1 levels in sham-operated and I/R injured mice with or without running-wheel exercise. (B) MALAT1 levels in I/R-runners treated with MALAT1 exogenous MALAT1 or siRNA MALAT1. Data are presented as the mean \pm standard deviation. siRNA, small interfering RNA; MALAT1, metastasis-associated lung adenocarcinoma transcript $1 ; \mathrm{I} / \mathrm{R}$, ischemia/reperfusion.

MALAT1 treatment enhanced the cognitive improvement induced by voluntary RW in I/R mice, whereas MALAT1 siRNA administration partially attenuated these effects. The I/R runners + MALAT1 group showed increase in the test duration, number of paths travelled and number of target crossings in the target quadrant when compared with the I/runners + MALAT1 blank group ( $\mathrm{P}<0.05$, Fig. 4). However, mice in I/R runners + MALAT1 siRNA groups revealed a reduction in the time spent, the number of paths travelled and the number of target crossings (vs. I/R runners + MALAT1 scrambled siRNA, $\mathrm{P}<0.05$, Fig. 4). The two control groups, I/R-runners + MALAT1 blank and I/R-runners + MALAT1 scrambled siRNA ones, showed no statistical difference $(\mathrm{P}>0.05)$.

Apoptosis induced by $I / R$ injury was reversed by $R W$. Voluntary RW had no effect on hippocampal cell apoptosis under sham operated conditions. The apoptotic cell proportion was increased in the hippocampus following $I / R$ injury ( $/ \mathrm{R}$ non-runners vs. sham non-runners, $\mathrm{P}<0.05$ ); however, significantly reduced in mice subjected to voluntary RW (I/R-runners vs. I/R non-runners, $\mathrm{P}<0.05$; Fig. 5). Administration of MALAT1 further reduced the number of apoptotic hippocampal cells in the I/R-runners (I/R-runners + MALAT1 vs. I/R-runners + MALAT1 blank, $\mathrm{P}<0.05)$. Conversely, MALAT1 siRNA treatment neutralized the effects of RW and increased the proportion of apoptotic cells 
A

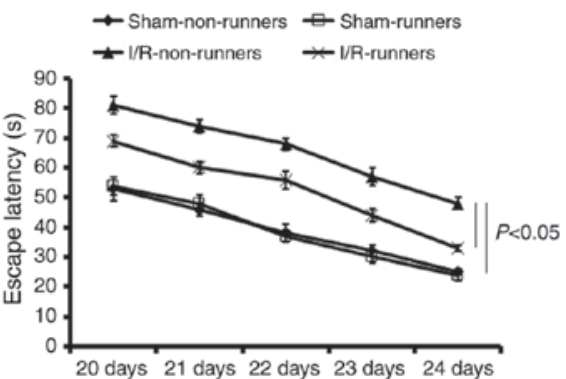

C Swimming traces

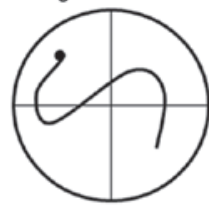

Sham-non-runners

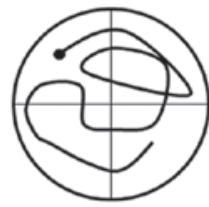

MALAT1 blank
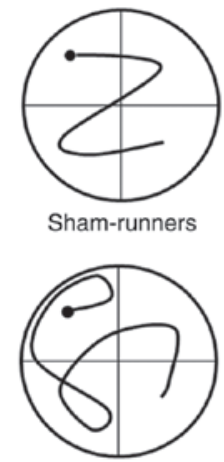

MALAT1
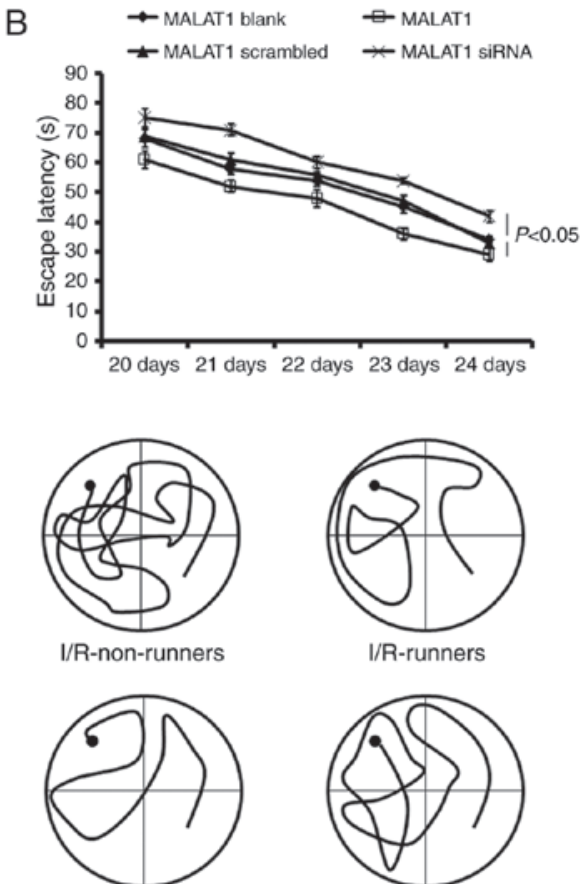

MALAT1 scambled

MALAT1 SIRNA

l/R-runners

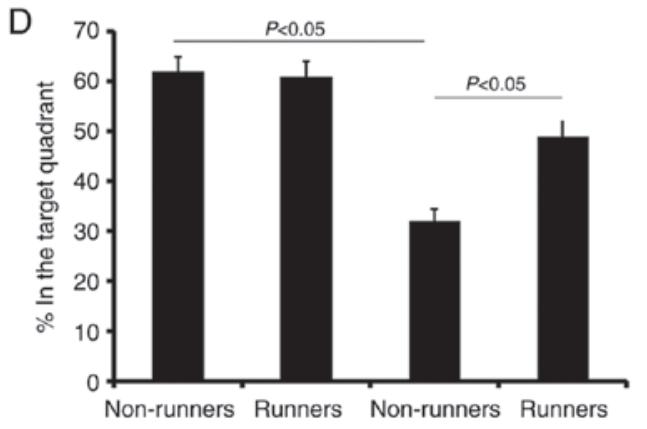

$\mathrm{E}$

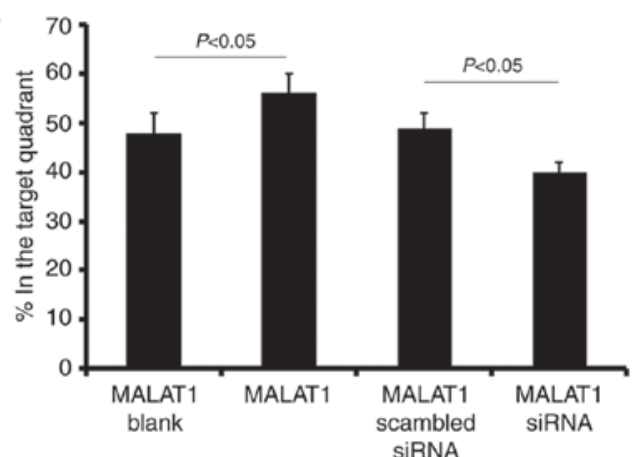

$\mathrm{F}$

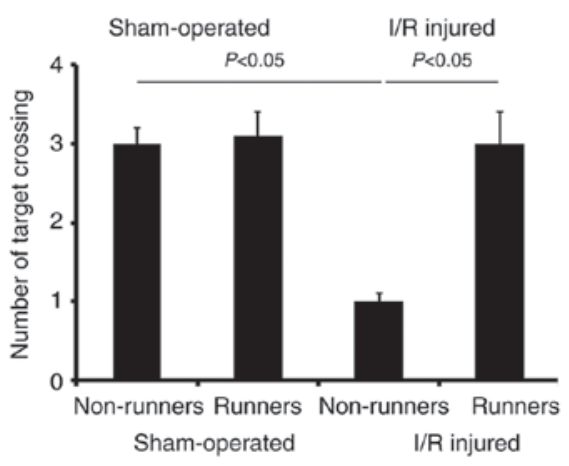

G

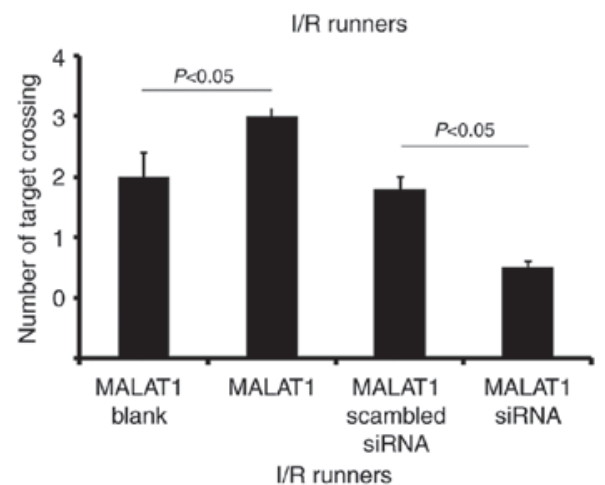

Figure 4. Learning and memory alterations assessed via the MWM test. The escape latency after hidden platform detected from 20 to 24 days post-operation. (A) Sham-operated and I/R injured mice with or without running wheel exercise. (B) I/R-runners treated with exogenous MALAT1 or siRNA MALAT1. (C) Representative swimming traces of different groups during the MWM test. (D and E) Percentages in the target quadrant of mice that underwent different treatments. (F and G) Number of target crossings of mice with different treatments. Data are presented as the mean \pm standard deviation. MWM, Morris Water Maze; siRNA, small interfering RNA; MALAT1, metastasis-associated lung adenocarcinoma transcript 1; I/R, ischemia/reperfusion.

(I/R-runners + MALAT1 siRNA vs. I/R-runners + MALAT1 scrambled siRNA, $\mathrm{P}<0.05)$.

Expression of apoptosis-associated proteins. Quantitative analysis was performed using western blotting. Following I/R injury, the expression levels of $\mathrm{Bax}, \mathrm{Bad}$, and caspase- $3 /-8$ were increased, whereas those of $\mathrm{Bcl}-2$ and $\mathrm{Bcl}-\mathrm{xL}$ were reduced (I/R non-runners vs. sham non-runners; $\mathrm{P}<0.05$; Fig. 6). RW exercise protected hippocampal cells from apoptosis. The present findings demonstrated that spontaneous RW 


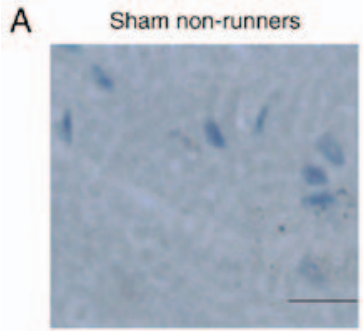

L/R-runners+ MALAT1 blank
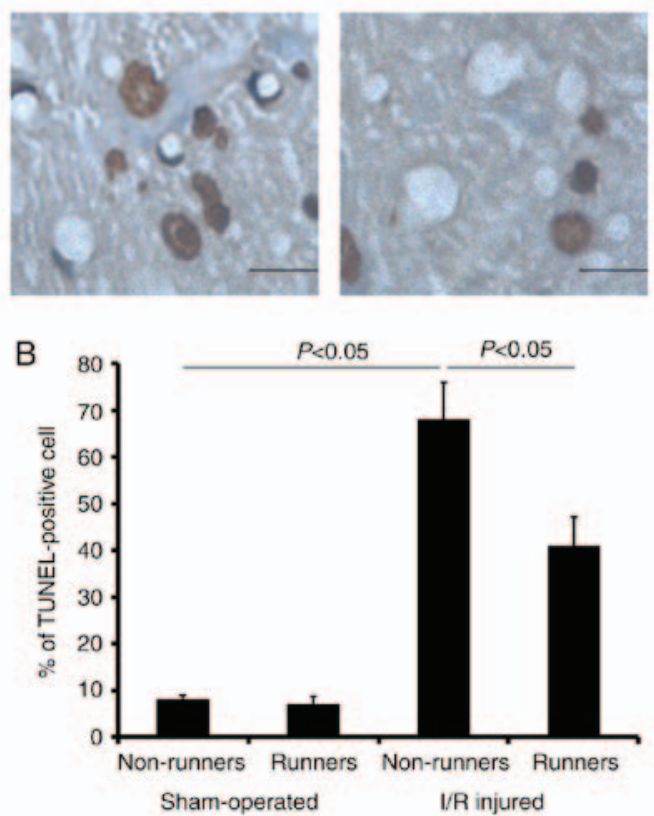

Sham-runners

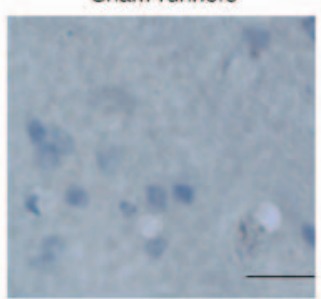

l/R-runners+

MALAT1

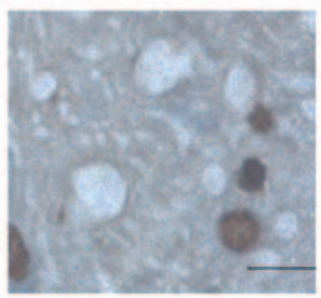

$\mathrm{C}$

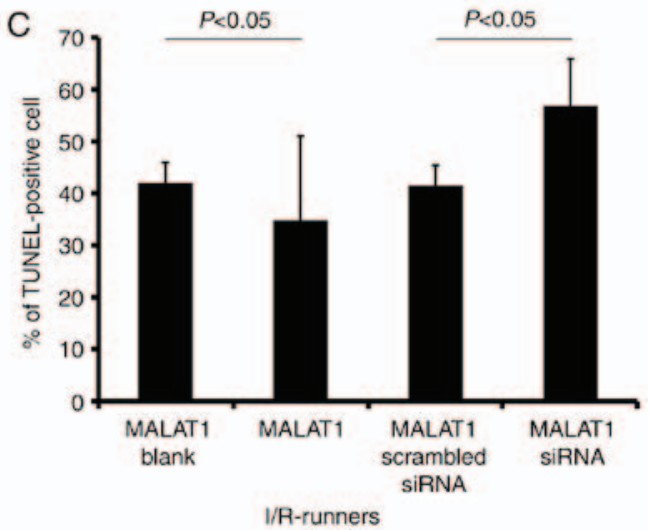

I/R-non-runners

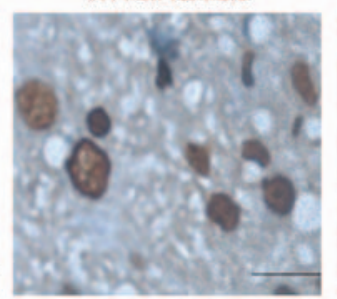

1/R-runnerS+MALAT1

scrambled siRNA
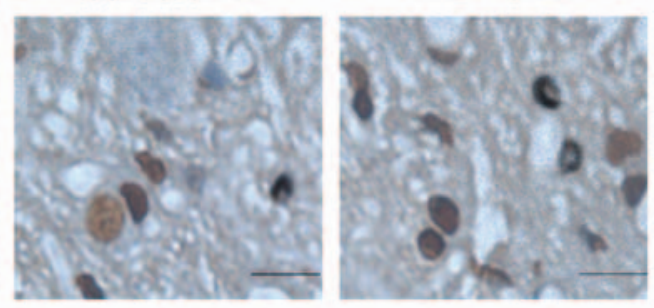

I/R-runners+

MALAT1 siRNA

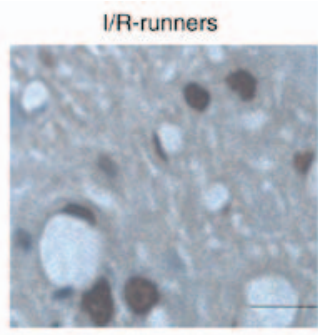
证
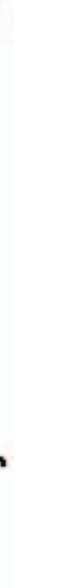

Figure 5. Apoptosis evaluation by TUNEL detections. (A) Representative apoptotic files detected in different groups. Scale bar, $2.5 \mu$ m. Percentages of TUNEL-positive cells were evaluated in the hippocampus of (B) runners and non-runners and (C) I/R-runners treated with exogenous MALAT1 or siRNA MALAT1. Data are presented as the mean \pm standard deviation. TUNEL, terminal deoxynucleotidyl transferase dUTP nick end labeling; siRNA, small interfering RNA; MALAT1, metastasis-associated lung adenocarcinoma transcript 1; I/R, ischemia/reperfusion.
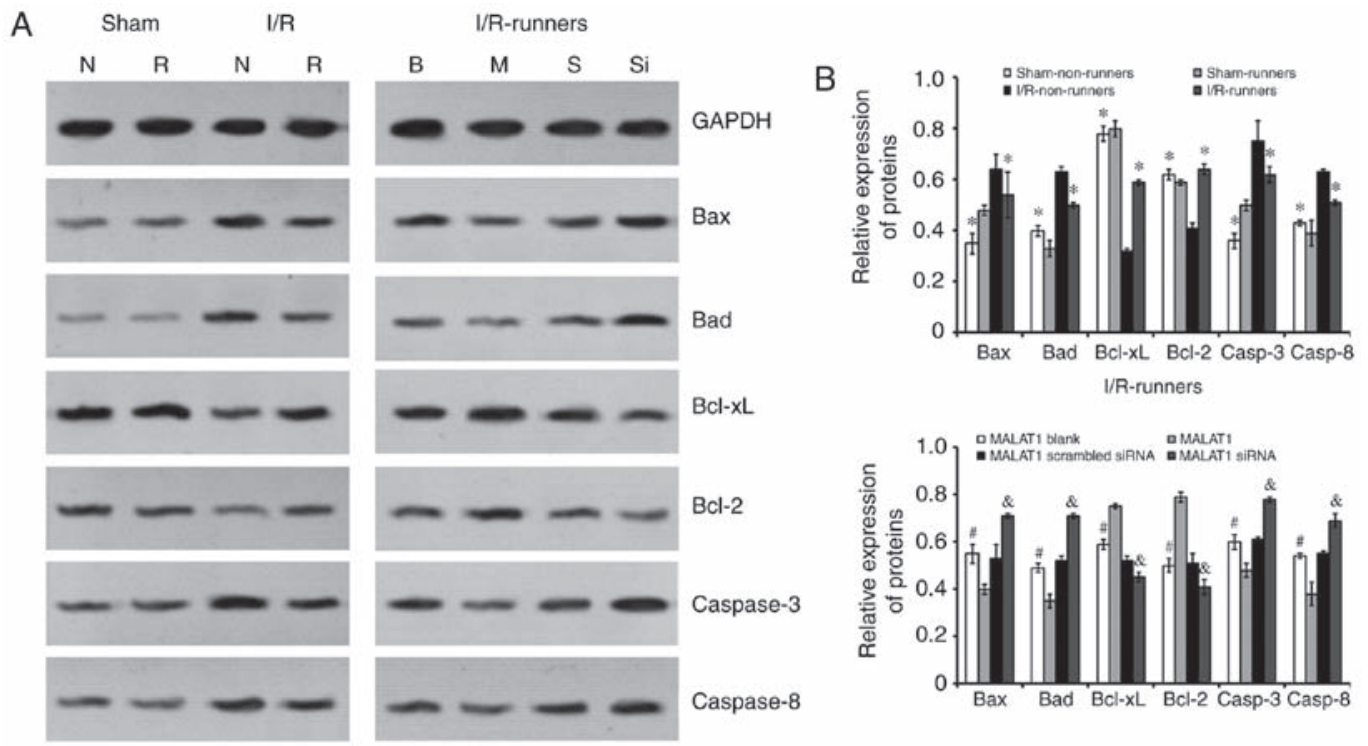

Figure 6. Expression of apoptosis-associated proteins in the hippocampus of mice from different groups. (A) Representative bands of the different proteins detected by western blotting. (B) Quantitative analysis of the apoptosis-associated proteins. "P<0.05 vs. I/R-Non-runners; "P<0.05 vs. MALAT1-treated; ${ }^{\&} \mathrm{P}<0.05$ vs. MALAT1 scrambled siRNA group. Data are presented as the mean \pm standard deviation. siRNA, small interfering RNA; MALAT1, metastasis-associated lung adenocarcinoma transcript 1; I/R, ischemia/reperfusion; B, MALAT1 Blank; M, MALAT1; S, MALAT1 scrambled siRNA; Si, MALAT1 siRNA; N, Non-runners; R, runners; Bcl-2, B cell leukemia/lymphoma 2; Bax, BCL2-associated X; Bad, BCL2 associated agonist of cell death; Bcl-xL, B-cell lymphoma-extra large. 
downregulated the expression of Bax, Bad and caspase-3/-8, whereas Bcl-2 and Bcl-xL expression levels were upregulated in the hippocampus following $\mathrm{I} / \mathrm{R}$ injury ( $\mathrm{I} / \mathrm{R}$-runners vs. I/R-Non-runners; $\mathrm{P}<0.05$; Fig. 6). Consistent with the TUNNEL staining results, MALAT1 treatment increased the inhibition of apoptosis induced by RW in I/R mice, whereas MALAT1 siRNA administration attenuated this effect. Specifically, the Bax, Bad, and caspase-3/-8 levels were reduced, whereas the $\mathrm{Bcl}-2$ and $\mathrm{Bcl}-\mathrm{xL}$ levels increased following MALAT1 treatment (I/R-runners + MALAT1 vs. I/R-runners + MALAT1 blank; P<0.05; Fig. 6). The expression levels of Bax, Bad, and caspase-3/-8 were partially upregulated and $\mathrm{Bcl}-2$ and Bcl-xL expression levels were reduced, by MALAT1 siRNA administration in I/R-runners (I/R-runners + MALAT1 siRNA vs. I/R-runners + MALAT1 scrambled siRNA; P<0.05). The expression of apoptosis-associated proteins tested in sham operated groups showed no significant difference following RW (sham non-runners vs. sham-runners, $\mathrm{P}<0.05$ ). There were also no significant differences identified between the MALAT1 treatment control groups.

\section{Discussion}

The present study aimed to investigate whether increased hippocampal MALAT1 expression induced spontaneous RW following I/R injury in mice, which may be associated with cognitive recovery. The current findings revealed that $I / R$ injury induced significant neurological and cognitive function deficits, as well as increased neuronal apoptosis in mice. Spontaneous RW partially restored this neurological and cognitive impairment and reduced hippocampal apoptosis. Simultaneously, the expression of MALAT1, an lncRNA demonstrated to be involved in cancer cell proliferation and metastasis was significantly restored following RW exercise. Specific siRNA targeting MALAT1 neutralized the neuroprotective effects induced by voluntary RW exercise, whereas MALAT1 administration enhanced these effects.

The pathological condition of cerebral ischemia is responsible for learning and memory impairments, and adversely affects the everyday and social lives of survivors (25). The present study revealed that I/R injury may lead to significant neurological loss, learning and memory deficits, which is consistent with previous findings $(4,26)$.

Previous studies have demonstrated that exercise has beneficial effects and promotes functional recovery in animal models of stroke $(27,28)$. Voluntary RW is reportedly the most valid treatment for promoting the recovery of motor function. Rats that underwent voluntary exercise also had a lower corticosterone stress response, compared with other trialed exercise paradigms (13). However, the underlying molecular mechanisms reported have been inconsistent and remain to be fully elucidated. The present study revealed that voluntary RW exercise partially reverses hippocampal MALAT1 expression, reduces infarction volume, decreases the hippocampal TUNEL-positive cell proportion and alters the expression of apoptosis-associated proteins. MALAT1 siRNA treatment neutralized the neuroprotective effects induced by voluntary RW exercise, whereas exogenous MALAT1 administration increased these protective effects. Therefore, the present study hypothesized that the voluntary RW exercise-induced recovery of cognitive functions may be associated with hippocampal apoptosis inhibition mediated by MALAT1.

Cerebral miRNA and piwi-interacting RNA profiles change extensively, these may be involved in neuroplasticity and the modulation of brain damage following ischemic injury. Miao et al (4) revealed that cortical and hippocampal miRNA expression alterations in $\mathrm{I} / \mathrm{R}$ mice were responsible for ischemic postconditioning-induced neuroprotection. Previous studies reported that ischemia also influences the lncRNA profile bioinformatics analysis identified a $>90 \%$ sequence homology between several stroke-responsive lncRNAs genes and protein-coding genes located on different chromosomes, indicating that these IncRNAs may be pseudogenes $(8,29,30)$. Many of the 'stroke-responsive' lncRNAs are homologous to protein-coding genes that are associated with ribosomal complex formation, splicing, translation initiation and the nuclear import of mRNAs. Therefore, they may stabilize those mRNAs in order to partially recover the protein generation inhibited by the stroke, during the acute phase following injury (8). Previous studies have determined that these 'stroke-responsive' IncRNAs may be involved in chromatin modifications, transcription factor activity and apoptosis regulation (31-33).

The present study revealed that the IncRNA MALAT1 level was significantly reduced following I/R injury and was partially restored by spontaneous RW exercise. MALAT1 siRNA treatment neutralized the neuroprotection induced by voluntary RW exercise, including cognitive recovery and apoptosis inhibition, whereas treatment with MALAT1 enhanced these effects.

MALAT1 is involved with the survival of various tumor types and may act as a valid prognostic biomarker for cancer (34). A previous study revealed its role in extensive biological processes. For example, MALAT1 lncRNA was demonstrated to protect human brain vascular endothelial cells from oxygen-glucose deprivation and re-oxygenation-induced apoptosis in vitro (22). The current study revealed that voluntary RW exercise setting protected I/R injured mice from the cognitive decline induced by MALAT1-regulated apoptosis inhibition.

The present study used TUNEL staining and protein level detection to identify hippocampal apoptosis following the application of different treatments. The apoptosis prevention-induced by voluntary RW following I/R injury might depend on endogenic MALAT1 regulation. Spontaneous RW downregulated Bax, Bad and caspase-3/-8, whereas Bcl-2 and $\mathrm{Bcl}-\mathrm{xL}$ were upregulated in the hippocampus of mice post I/R injury. Those expressional alterations were partially reversed by MALAT1 siRNA treatment and enhanced by the administration of exogenous MALAT1. Apoptosis, a complex biochemical process, is influenced by a variety of factors $(35,36)$. It has been previously established that the Bcl-2 family functions as inhibitors (such as Bcl-2 and Bcl-xL) or promotors (such as Bax, Bcl-xS, Bad, and Bak) during cell apoptosis. Caspase-3 activation is considered a key mechanism of apoptosis $(37,38)$. Therefore, the current findings indicate that voluntary RW may inhibit the damage caused by apoptosis with MALAT1 regulation, in I/R mice.

In conclusion, the present study demonstrated that $I / R$ injury induced significant neurological deficits and cognitive impairments, as well as increased neuronal apoptosis in mice. Spontaneous RW exercise may partially restore these 
impairments, reduce hippocampal apoptosis and alter the expression of apoptosis-associated proteins. siRNA targeting MALAT1 neutralized the neuroprotective effects induced by voluntary RW exercise, whereas exogenous MALAT1 enhanced these effects. The present findings suggested that spontaneous RW exercise had a neuroprotective role, via MALAT1 regulation, following $\mathrm{I} / \mathrm{R}$ injury in mice.

\section{Competing interests}

The authors declare that they have no competing interests.

\section{References}

1. Durukan A and Tatlisumak T: Preconditioning-induced ischemic tolerance: A window into endogenous gearing for cerebroprotection. Exp Transl Stroke Med 2: 2, 2010.

2. Zhao H, Sapolsky RM and Steinberg GK: Interrupting reperfusion as a stroke therapy: Ischemic postconditioning reduces infarct size after focal ischemia in rats. J Cereb Blood Flow Metab 26: 1114-1121, 2006.

3. Lin XM, Zhang ZY, Wang LF, Zhang L, Liu Y, Liu XL, Yang XC, Cui $\mathrm{L}$ and Zhang L: Attenuation of tumor necrosis factor-alpha elevation and improved heart function by postconditioning for $60 \mathrm{sec}$ in patients with acute myocardial infarction. Chin Med 123: 1833-1839, 2010.

4. Miao W, Bao TH, Han JH, Yin M, Zhang J, Yan Y and Zhu YH: Neuroprotection induced by post-conditioning following ischemia/reperfusion in mice is associated with altered microRNA expression. Mol Med Rep 14: 2582-2588, 2016.

5. Niland CN, Merry CR and Khalil AM: Emerging roles for long non-coding RNAs in cancer and neurological disorders. Front Genet 3: 25, 2012.

6. Huttenhofer A, Schattner P and Polacek N: Non-coding RNAs: Hope or hype? Trends Genet 21: 289-297, 2005.

7. Zhang Q, Matsuura K, Kleiner DE, Zamboni F, Alter HJ and Farci P: Analysis of long noncoding RNA expression in hepatocellular carcinoma of different viral etiology. J Transl Med 14: 328, 2016.

8. Dharap A, Nakka VP and Vemuganti R: Effect of focal ischemia on long noncoding RNAs. Stroke 43: 2800-2802, 2012.

9. Puthanveetil P, Chen S, Feng B, Gautam A and Chakrabarti S: Long non-coding RNA MALAT1 regulates hyperglycaemia induced inflammatory process in the endothelial cells. J Cell Mol Med 19: 1418-1425, 2015.

10. Michalik KM, You X, Manavski Y, Doddaballapur A, Zörnig M, Braun T, John D, Ponomareva Y, Chen W, Uchida S, et al: Long noncoding RNA MALAT1 regulates endothelial cell function and vessel growth. Circ Res 114: 1389-1397, 2014.

11. Thum $\mathrm{T}$ and Fiedler J: LINCing MALAT1 and angiogenesis. Circ Res 114: 1366-1368, 2014.

12. Hu T, Zhou FJ, Chang YF, Li YS, Liu GC, Hong Y, Chen HL, Xiyang YB and Bao TH: miR21 is associated with the cognitive improvement following voluntary running wheel exercise in TBI mice. J Mol Neurosci 57: 114-122, 2015.

13. Ke Z, Yip SP, Li L, Zheng XX and Tong KY: The effects of voluntary, involuntary, and forced exercises on brain-derived neurotrophic factor and motor function recovery: A rat brain ischemia model. PLoS One 6: e16643, 2011.

14. Bao TH, Miao W, Han JH, Yin M, Yan Y, Wang WW and Zhu YH: Spontaneous running wheel improves cognitive functions of mouse associated with miRNA expressional alteration in hippocampus following traumatic brain injury. J Mol Neurosci 54: 622-629, 2014.

15. XiYang YB, Wang YC, Zhao Y, Ru J, Lu BT, Zhang YN, Wang NC, Hu WY, Liu J, Yang JW, et al: Sodium channel voltage-gated beta 2 plays a vital role in brain aging associated with synaptic plasticity and expression of COX5A and FGF-2. Mol Neurobiol 53: 955-967, 2016.

16. Xiong X, Gu L, Zhang H, Xu B, Zhu S and Zhao H: The protective effects of $\mathrm{T}$ cell deficiency against brain injury are ischemic model-dependent in rats. Neurochem Int 62: 265-270, 2013.

17. Joo SP, Xie W, Xiong X, Xu B and Zhao H: Ischemic postconditioning protects against focal cerebral ischemia by inhibiting brain inflammation while attenuating peripheral lymphopenia in mice. Neuroscience 23: 149-157, 2013.
18. Hill JK, Gunion-RinkerL,Kulhanek D, Lessov N,Kim S,Clark WM, Dixon MP, Nishi R, Stenzel-Poore MP and Eckenstein FP: Temporal modulation of cytokine expression following focal cerebral ischemia in mice. Brain Res 820: 45-54, 1999.

19. Loane DJ, Pocivavsek A, Moussa CE, Thompson R, Matsuoka Y, Faden AI, Rebeck GW and Burns MP: Amyloid precursor protein secretases as therapeutic targets for traumatic brain injury. Nat Med 15: 377-379, 2009

20. Gong G, Xiang L, Yuan L, Hu L, Wu W, Cai L, Yin L and Dong H: Protective effect of glycyrrhizin, a direct HMGB1 inhibitor, on focal cerebral ischemia/reperfusion-induced inflammation, oxidative stress, and apoptosis in rats. PLoS One 9: e89450, 2014.

21. Livak KJ and Schmittgen TD: Analysis of relative gene expression data using real-time quantitative PCR and the $2^{-\Delta \Delta C \mathrm{~T}}$ method. Methods 25: 402-408, 2001.

22. Xin JW and Jiang YG: Long noncoding RNA MALAT1 inhibits apoptosis induced by oxygen-glucose deprivation and reoxygenation in human brain microvascular endothelial cells. Exp Ther Med 13: 1225-1234, 2017.

23. Kuete V, Sandjo LP, Mbaveng AT, Seukep JA, Ngadjui BT and Efferth T: Cytotoxicity of selected Cameroonian medicinal plants and Nauclea pobeguinii towards multi-factorial drug-resistant cancer cells. BMC Complement Altern Med 15: 309, 2015.

24. Skup M, Dwornik A, Macias M, Suleczak D, Wiater M and Czarkoeska-Bauch J: Long-term locomotor training upregulates TrkB(FL) receptor-like proteins, brain-derived neurotrophic factor, and neurotrophin 4 with different topographies of expression in oligodendroglia and neurons in the spinal cord. Exp Neurol 176: 289-307, 2002

25. Vaughan L, Bushnell C, Bell CL and Espeland MA: Global cognitive function before, surrounding, andafter ischemic stroke: The role of risk and protective factors varies with time among ischemic stroke survivors. Neuropsychol Dev Cogn B Aging Neuropsychol Cogn 23: 117-131, 2016

26. Moghimi M, Parvardeh S, Zanjani TM and Ghafghazi S: Protective effect of $\alpha$-terpineol against impairment of hippocampal synaptic plasticity and spatial memory following transient cerebral ischemia in rats. Iran J Basic Med Sci 19: 960-969, 2016.

27. DeBow SB, Davies MA, Clarke HL and Colbourne F: Constraint-induced movement therapy and rehabilitation exercises lessen motor deficits and volume of brain injury after striatal hemorrhagic stroke in rats. Stroke 34: 1021-1026, 2003.

28. Burnett MG, Shimazu T, Szabados T, Muramatsu H, Detre JA and Greenberg JH: Electrical forepaw stimulation during reversible forebrain ischemia decreases infarct volume. Stroke 37: 1327-1331, 2006.

29. Dharap A, Nakka VP and Vemuganti R: Altered expression of PIWI RNA in the rat brain after transient focal ischemia. Stroke 42: 1105-1109, 2011.

30. Hirotsune S, Yoshida N, Chen A, Garrett L, Sugiyama F, Takahashi S, Yagami K, Wynshaw-Boris A and Yoshiki A: An expressed pseudogene regulates the messenger-RNA stability of its homologous coding gene. Nature 423: 91-96, 2003.

31. Gupta RA, Shah N, Wang KC, Kim J, Horlings HM, Wong DJ, Tsai MC, Hung T, Argani P, Rinn JL, et al: Long non-coding RNA HOTAIR reprograms chromatin state to promote cancer metastasis. Nature 464: 1071-1076, 2010.

32. Huarte M, Guttman M, Feldser D, Garber M, Koziol MJ Kenzelmann-Broz D, Khalil AM, Zuk O, Amit I, Rabani M, et al: A largeintergenic noncoding RNA induced by p53 mediates global gene repression in the 553 response. Cell 142: 409-419, 2010.

33. Zhang J, Yuan L, Zhang X, Hamblin MH, Zhu T, Meng F, Li Y, Chen YE and Yin KJ: Altered long non-coding RNA transcriptomic profiles in brain microvascular endothelium after cerebral ischemia. Exp Neurol 277: 162-170, 2016.

34. Yan J, Zhou X, Dang Y, Yin C and Zhang G: Prognostic role of the long non-coding RNA metastasis-associated lung adenocarcinoma transcript 1 in various cancers: A meta-analysis. Mol Clin Oncol 4: 100-106, 2016.

35. Vaux D and Korsmeyer S: Cell death in development. Cell 96: 245-254, 1999.

36. Adams JM and Cory S: The Bcl-2 protein family: Arbiters of cell survival. Science 281: 1322-1326, 1998 .

37. Korsmeyer SJ: BCL-2 gene family and the regulation of programmed cell death. Cancer Res 59 (7 Suppl): S1693-S1700, 1999.

38. Cohen GM: Caspases: The executioners of apoptosis. Biochem J 326: 1-16, 1997.

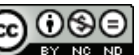

This work is licensed under a Creative Commons Attribution-NonCommercial-NoDerivatives 4.0 International (CC BY-NC-ND 4.0) License. 